\title{
APLICAÇÃO DAS TÉCNICAS DE ENGENHARIA DE MÉTODOS: ESTUDO DE CASO EM UMA EMPRESA DE CONFECÇÃO DE ROUPAS
}

\author{
Hedivigem Luana Rodrigues da Silva (UFCG/CDSA) hedyluana884@gmail.com \\ Jordana de Souza Nogueira (UFCG/CDSA) souza.s2.jordana@gmail.com \\ Júlio Cesar Vasconcelos Conserva (UFCG/CDSA) julio.conserva@ outlook.com \\ Manoel Cardoso Costa Segundo (UFCG/CDSA) manoelcardosocosta@ hotmail.com
}

\section{Resumo}

A partir do crescimento da indústria têxtil nacional e da identificação da necessidade das empresas do setor em conquistar um melhor desempenho em seus processos produtivos afim de obter a consolidação e vantagem competitiva, o presente artigo propõe a aplicação das técnicas de engenharia de métodos, realizando um estudo de caso e analise dos resultados para auxiliar a empresa estudada nesse desafio. Com isso, para atingir o objetivo de colaborar no alcance da melhoria do processo e consequentemente da alta produtividade, realizou-se um estudo de tempos e movimentos no processo de embalagem de camisetas no empreendimento de produção têxtil em questão, conseguindo organizar racionalmente o trabalho através da observação. Diante dos resultados obtidos constatou-se a oportunidade de melhoria no processo, fazendo-se assim o aconselhamento a empresa analisada.

Palavras-Chaves: (Engenharia de Métodos, Tempos e Métodos, Melhoria, Indústria têxtil)

\section{Introdução}

Tendo em vista o processo de fortalecimento da indústria têxtil no Brasil, que é atualmente impulsionada pelo crescimento do setor no agreste de Pernambuco e regiões próximas, as empresas do ramo tem a necessidade de dar respostas rápidas aos seus clientes para se consolidar no mercado.

Essa região é considerada a segunda maior produtora nacional, o conhecido polo da moda vem gerando bastante dinheiro para os envolvidos e muitos empregos, de acordo com a AD DIPER (Agência de Desenvolvimento Econômico de Pernambuco) a cadeia produtiva têxtil de Pernambuco movimenta mais de $\mathrm{R} \$ 5,6$ bilhões por ano e segundo a pesquisa realizada pela Consultoria IEMI em 2017 no estado é produzido cerca de 225 milhões de peças anualmente. 
Neste contexto, as empresas são impulsionadas pelo desejo de inovação buscando novas formas de atender seus clientes, com isso o mercado vem procurando constantemente técnicas mais rápidas e práticas de produção tendo que ampliar suas redes de aprendizagem e criar métodos inovadores para o crescimento do negócio.

Com esse cenário, evidenciasse que o uso de técnicas que venham a ajudar as organizações nesse desafio de ser mais eficiente são de grande relevância para seu sucesso. Assim o uso do estudo de tempos e movimentos mostrasse um importante e essencial aliado das empresas que procuram um diferencial, permitindo o aprimoramento sem a necessidade de altos investimentos a partir do aperfeiçoamento das técnicas de trabalho levando ao aumento da capacidade prática, fornecendo ainda informações para a tomada de decisão.

Desta forma, este artigo propõe a análise do estudo de caso com o uso das abordagens da engenharia de métodos na embalagem de vestuários com o propósito de auxiliar na inovação do processo produtivo de uma empresa do ramo de produção têxtil situada na cidade de CongoPB que faz parte das regiões próximas aos polos têxtil do agreste de Pernambuco, a fim de explorar os resultados através de técnicas que podem proporcionar melhoria da qualidade e produtividade dentro deste setor.

\section{Referencial teórico}

\subsection{Indústria têxtil}

A indústria têxtil do Brasil nasceu ainda no período colonial, desenvolveu-se acentuadamente a partir do início do século XX e encontrou a maturidade na década de 1940, quando chegou a ser considerada um setor industrial dinâmico de uma economia subdesenvolvida. Isto se verificou tendo em vista ter alcançado uma sólida estrutura que lhe conferiu a posição de segundo lugar na produção têxtil mundial, e de exportar para grande parte do mundo, por ocasião da Segunda Guerra Mundial (KON; COAN, 2009).

A indústria têxtil e do vestuário encontra-se em época de grandes mudanças, o mundo em que vivemos fortemente marcados pela revolução das comunicações das últimas décadas mudou substancialmente e exige uma formação profissional em sintonia com a nova ordem internacional que vem se desenvolvendo de uma forma muito dinâmica (MELO, 2007 apud MOURA; CÍRICO, 2019). 
Segundo Fujita e Jorente (2015) a análise da trajetória da indústria têxtil brasileira demonstra que existe um potencial de criação e de inovação a ser explorado que necessita de investimento em tecnologia como fator de mudança tendo em vista que a sociedade brasileira demonstra uma diversidade cada vez mais segmentada em suas classes sociais.

Pensando em novos métodos de gestão e em novas tecnologias a orientação só pode ser uma, o investimento em modernização. Um dos possíveis caminhos a seguir é a utilização crescente de sistemas de produção flexíveis para responder rapidamente a segmentos do mercado onde o valor acrescentado é elevado. Desta forma é válido ressaltar que o investimento em melhorias nos equipamentos a fim de reduzir o tempo de produção por peça é mais um passo para tornar a organização mais eficiente e condizente com a atual realidade das indústrias (ARAÚJO, 1996 apud MOURA; CÍRICO, 2019).

\subsection{Estudo de tempos e métodos}

A mensuração do trabalho, feita de forma científica, utilizando técnicas estatísticas, teve seu início na primeira metade do século passado, e era aplicada apenas em organizações do tipo industrial. Seus precursores foram Frederick W. Taylor e o casal Frank e Lílian Gilbreth. O objetivo da medida dos tempos de trabalho era determinar a melhor e mais eficiente forma de desenvolver uma tarefa específica (PEINADO; GRAEML, 2007).

O estudo de movimentos, e de tempos é o estudo sistemático dos sistemas de trabalho com os seguintes objetivos: (1) desenvolver o sistema e o método preferido, usualmente aquele de menor custo; (2) padronizar esse sistema e método; (3) determinar o tempo gasto por uma pessoa qualificada e devidamente treinada, trabalhando num ritmo normal, para executar uma tarefa ou operação específica; e (4) orientar o treinamento do trabalhador no método preferido (BARNES, 1977).

Peinado e Graeml (2007) o estudo de tempos, movimentos e métodos aborda técnicas que submetem a uma detalhada análise de cada operação de uma dada tarefa, com o objetivo de eliminar qualquer elemento desnecessário à operação e determinar o melhor e mais eficiente método para executá-la.

\subsubsection{Fluxograma}

Fluxogramas são formas de representar, por meio de símbolos gráficos, a sequência dos passos de um trabalho para facilitar sua análise. Um fluxograma é um recurso visual utilizado pelos 
gerentes de produção para analisar sistemas produtivos, buscando identificar oportunidades de melhorar a eficiência dos processos. O fluxograma permite rápida visualização e entendimento (PEINADO; GRAEML, 2007).

Normalmente são utilizados apenas cinco símbolos para descrever tais processos:

Quadro 1 - Simbologia de fluxograma

\begin{tabular}{|c|l|}
\hline SÍMBOLO & \multicolumn{1}{c|}{ DESCRIÇÃo } \\
\hline $\mathbf{O}$ & $\begin{array}{l}\text { Operação: ocorre quando se modifica intencionalmente um objeto em } \\
\text { qualquer de suas características fisicas ou químicas, ou também quando } \\
\text { se monta ou desmontam componentes e partes. }\end{array}$ \\
\hline$\square$ & $\begin{array}{l}\text { Transporte: ocorre quando um objeto ou matéria-prima é transferido de } \\
\text { um lugar para o outro, de uma seção para outra, de um prédio para } \\
\text { outro. }\end{array}$ \\
\hline$\square$ & $\begin{array}{l}\text { Espera: Ocorre quando um objeto ou matéria prima é colocado } \\
\text { intencionalmente numa posição estática. O material permanece } \\
\text { aguardando processamento ou encaminhamento. }\end{array}$ \\
\hline$\square$ & $\begin{array}{l}\text { Inspeção: ocorre quando um objeto ou matéria-prima é examinado para } \\
\text { sua identificação, quantidade ou condição de qualidade. }\end{array}$ \\
\hline$\nabla$ & $\begin{array}{l}\text { Armazenagem: ocorre quando um objeto ou matéria-prima é mantido } \\
\text { em área protegida específica na forma de estoque. }\end{array}$ \\
\hline
\end{tabular}

Fonte: Adaptado de Peinado e Graeml (2007)

\subsubsection{Números de ciclos a serem cronometrados}

O tempo requerido à execução dos elementos de uma operação varia ligeiramente de ciclo para ciclo. Mesmo que o operador trabalhe a um ritmo constante, nem sempre executará cada elemento de ciclos consecutivos exatamente no mesmo tempo. O estudo de tempos um processo de amostragem; consequentemente, quanto maior o número de ciclos cronometrado tanto mais representativos serão os resultados obtidos para a atividade em estudo, mensurado na fórmula (BARNES, 1977).

Segundo Peinado e Graeml (2007), é necessário utilizar um cálculo estatístico de determinação do número de observações, mensurado na fórmula.

$$
N=\left(\frac{Z c \cdot R}{\operatorname{Er} \cdot D 2 \cdot \bar{X}}\right)^{2}
$$

Onde,

$\mathrm{N}$ = número de operações a serem cronometrados 
$\mathrm{Z}=$ coeficiente de distribuição normal para uma probabilidade determinada

$\mathrm{R}=$ amplitude da amostra

$\mathrm{Er}=$ erro relativo da medida

$\mathrm{d}_{2}=$ coeficiente em função do número de cronometragem realizadas preliminarmente

$\bar{x}=$ média dos valores das observações

\subsubsection{Tempo normal e tempo padrão}

A velocidade do operador é determinada subjetivamente pelo cronoanalista. Para a velocidade de operação normal do operador é atribuída uma taxa de velocidade, ou ritmo, de $100 \%$. Velocidades acima do normal apresentam valores superiores a $100 \%$ e velocidades abaixo do normal apresentam valores inferiores a 100\% (PEINADO; GRAEML, 2007)

Ainda de acordo com Peinado e Graeml (2007) para tornar o tempo utilizável para todos os trabalhadores, a medida da velocidade, que é expressa como uma taxa de desempenho que reflete o nível de esforço do operador observado deve também ser incluída para "normalizar" o trabalho, descrita na fórmula.

$$
T N=T C x V
$$

Após ser determinado o TN calcula-se o tempo padrão TP. Segundo Peinado e Graeml (2007) o tempo padrão é calculado multiplicando-se o tempo normal por um fator de tolerância para compensar o período que o trabalhador, efetivamente, não trabalha, mensurado na fórmula.

$$
T P=T N x F T
$$

De acordo com Barnes (1977), não é de se esperar que uma pessoa trabalhe o dia inteiro sem algumas interrupções; o operador pode dispender o seu tempo em necessidades pessoais, descansando ou por motivos fora de seu controle. As tolerâncias para essas interrupções da produção podem ser classificadas em 1) tolerância pessoal, 2) tolerância para a fadiga, ou 3) tolerância de espera. Se estas três tolerâncias forem aplicadas uniformemente a todos os elementos, elas podem ser somadas e aplicadas juntas, necessitando um único cálculo.

$$
F T=\frac{T}{T-p}
$$

\section{Metodologia}

A princípio, foi feito um levantamento bibliográfico visando o embasamento teórico necessário para o estudo de tempos e métodos. Posteriormente e com toda a base teórica já adquirida, um 
estudo de caso foi conduzido para avaliar o processo de embalagem de camisetas em uma empresa de confecção têxtil de pequeno porte situada na cidade do Congo - PB.

De acordo com Yin (2001), um estudo de caso é uma investigação empírica que investiga um fenômeno contemporâneo dentro do seu contexto da vida real, especialmente quando os limites entre o fenômeno e o contexto não estão claramente definidos.

A metodologia da pesquisa configura-se aplicada, quanto a natureza, pois objetiva gerar conhecimentos para aplicação prática e dirigidos à solução de problemas específicos. Envolve verdades e interesses locais. (SILVA, 2005). Já de acordo com o caráter qualitativo, a pesquisa é responsável pelo estudo do uso e coleta de uma variedade de materiais empíricos sobre a égide de métodos como estudo de caso, experiência pessoal, [...] responsáveis pela descrição dos momentos e significados da vida dos indivíduos (COOPER \& SCHINDLER, 2011).

Figura 1 - Processo de desenvolvimento do estudo

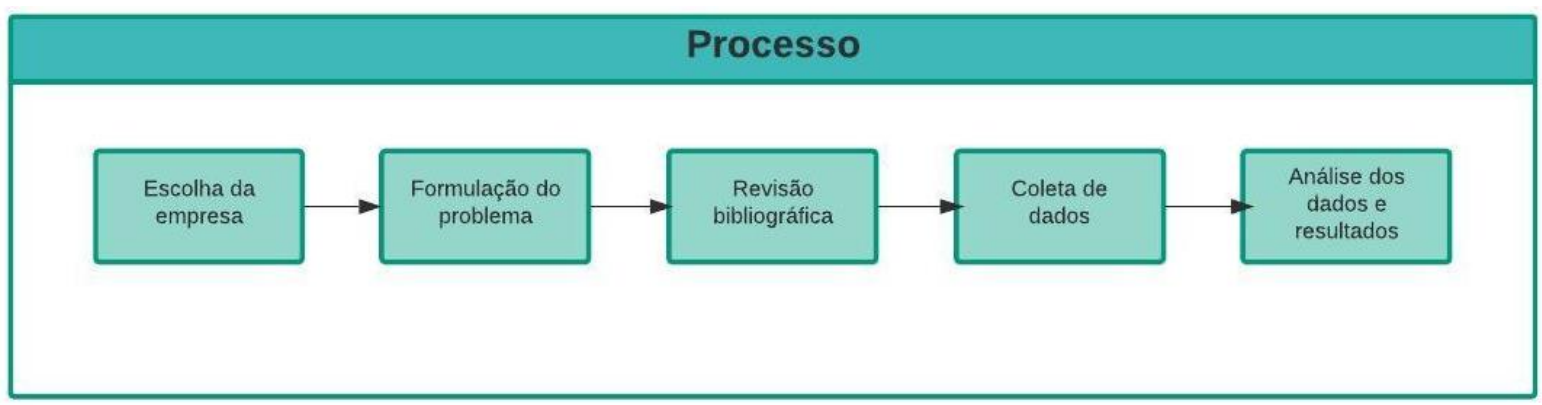

Fonte: Adaptado de Lima e Silva (2019)

Acima estão apresentadas as seguintes etapas:

- Escolha da Empresa: Um dos critérios dispostos foi o de que a empresa deveria conceder fácil acesso ao seu processo produtivo e que a presença da equipe de avaliação não influenciasse no mesmo;

- Formulação do problema: Se deu a partir da observação do processo e percepção da variabilidade do tempo de execução;

- Revisão Bibliográfica: Os conceitos de tempos e métodos como cronoanálise, Tempo Normal, Tempo Padrão e Fator de Tolerância com fins de embasamento teórico foram decisivos para o desenvolvimento desse estudo;

- Coleta de dados: Realizou-se por meio de cronometragem utilizando Smartphone e também por meio de entrevistas semiestruturadas; 
- Análise dos dados e resultados: Por fim, observou-se os dados obtidos no processo de embalagem de peças e na cronometragem de etapas desse processo, de modo a obter o Número de ciclos, o Tempo Normal e o Tempo Padrão.

\section{Resultados}

\subsection{Processo de embalagem das peças}

A partir da observação na empresa da execução da atividade de embalagem foi possível representar seus processos por meio da construção do fluxograma a seguir:

Figura 2 - Fluxograma do processo de embalagem das peças

Descrição
Peças no saco
Abrir peça na mesa verificando falhas
Pegar aplicador e tag do saco
Colocar tag na peça
Dobrar a peça
Abrir embalagem
Colocar peça na embalagem
Colocar peça embalada no saco excesso de ar

Fonte: Autoria própria (2020) 
A partir da análise do fluxograma elaborado pode-se observar que o processo é dividido em 12 micro processos, e para facilitar o estudo de tempos e métodos podem ser divididos em 4 principais: abertura, colocação de tags, dobrar e colocar na bolsa.

\subsection{Cronometragens}

Na tabela a seguir expõe-se os tempos obtidos durante o estudo, sendo realizado 10 medições de tempo sendo divididas em 4 processos.

Tabela 1 - Cronometragens

\begin{tabular}{cccccc}
\hline Medição & Abertura & Colocação de tags & Dobra & Colocar na bolsa & Total \\
\hline 1 & 5,29 & 5,55 & 9,14 & 18,74 & 38,72 \\
2 & 8,82 & 4,41 & 9,38 & 22,17 & 44,78 \\
3 & 5,64 & 6,17 & 7,13 & 15,96 & 34,9 \\
4 & 7,58 & 5,8 & 8,39 & 18,82 & 40,59 \\
5 & 5,94 & 6,97 & 8,84 & 22,18 & 43,93 \\
6 & 5,57 & 5,07 & 7,78 & 20,64 & 39,06 \\
7 & 7,33 & 5,91 & 8,29 & 19,9 & 41,43 \\
8 & 8,19 & 6,53 & 9,23 & 19,56 & 43,51 \\
9 & 8,5 & 5,51 & 7,1 & 17,31 & 38,42 \\
10 & 6,16 & 6,97 & 9,57 & 18,18 & 40,88 \\
\hline Média & $\mathbf{6 , 9 0}$ & $\mathbf{5 , 8 9}$ & $\mathbf{8 , 4 8}$ & $\mathbf{1 9 , 3 4}$ & $\mathbf{4 0 , 6 2}$ \\
\hline
\end{tabular}

Fonte: Autoria própria (2020)

Podemos observar a partir dos dados que a atividade estudada teve como tempo cronometrado (TC) 40,62 segundos em média e que o tempo total varia bastante nas 10 amostras, apresentando assim uma amplitude de amostra (R) de 9,88 que pode ser explicado pelo grande número de distrações que o operador demonstrou.

\subsection{Número de ciclos}

Para podermos observar a partir da expressão a seguir, o número mínimo de amostras fundamental para a realização do estudo, utilizando-se 10 cronometragens para amostra inicial, considerando um grau de confiança de $95 \%$ e erro relativo de $5 \%$ : 


$$
N=\left(\frac{Z c \cdot R}{\operatorname{Er} \cdot D 2 \cdot \bar{X}}\right)^{2}
$$

Onde:

$\mathrm{Zc}=1,96$

$\mathrm{D} 2=3,078$

$\mathrm{Er}=0,05$

$\bar{X}=40,62 \mathrm{~s}$

$\mathrm{R}=9,88$

Então:

$$
\begin{gathered}
N=\left(\frac{1,96 \cdot 9,88}{0,05 \cdot 3,078 \cdot 40,62}\right)^{2} \\
N=9,59
\end{gathered}
$$

Com isso, o número mínimo de cronometragens para realização do estudo é de 10 amostras.

\subsection{Tempo normal}

Para realização do cálculo do tempo normal (TN) foi necessário estimar a velocidade (V) do operador, e após observação da atividade se determinou a mesma como sendo 90\%, pois apesar de demonstrar experiência na execução o operador dispõe de diversas distrações como TV e conversas com outras pessoas que por sua vez influenciam diretamente no seu ritmo de trabalho.

$$
\begin{gathered}
\boldsymbol{T N}=\boldsymbol{T C} \boldsymbol{x} \boldsymbol{V} \\
T N=40,62 \times 0,9 \\
T N=36,56 s
\end{gathered}
$$

\subsection{Tempo padrão}

Com o intuito de calcular o tempo padrão da atividade é primordial que se determine antes o fator de tolerância. Para isso, observou-se que o trabalhador cumpre 8 horas de trabalho de quinta a sábado, onde 20 min são para descanso e 15 min para necessidades pessoais.

$$
F T=\frac{T}{T-p}
$$




$$
\begin{gathered}
F T=\frac{28000}{28000-1200-900} \\
F T=1,0786
\end{gathered}
$$

Com isso, é possível calcular o tempo padrão (TP) da atividade em estudo tendo como base o tempo normal e o fator de tolerância (FT) calculados anteriormente.

$$
\begin{gathered}
\boldsymbol{T P}=\boldsymbol{T N} \boldsymbol{x} \boldsymbol{F T} \\
T P=36,56 \times 1,0786 \\
T P=39,43 \mathrm{~s}
\end{gathered}
$$

\subsection{Análise dos resultados}

A partir da análise dos dados obtidos pelas amostras coletadas é possível observar que os mesmos apresentam uma amplitude de amostra que pode ser considerada alta quando se leva em consideração que se trata de uma atividade com curto tempo de execução. Apesar da operação apresentar um certo grau de padronização e o operador demonstrar experiência, essa disparidade apresentada nos resultados pode ser explicada pela presença de várias e constantes distrações no ambiente de trabalho analisado. Dentre as principais causas dessa diferença podemos citar:

- Falta de um espaço adequado para o operador;

- Retrabalho ocasionado por falhas nos processos anteriores (retirada de pontas de linhas);

- Uso constante da TV durante a execução da atividade;

- Conversas paralelas.

\subsection{Proposta de melhorias}

Após a realização do estudo pode-se identificar alguns elementos que impactam na produtividade da atividade analisada na empresa. Desse modo, foi sugerido que a empresa disponibilizasse uma mesa maior, para que o operador possa dispor ao seu alcance todos os itens necessários para sua tarefa, evitando que o mesmo precise fazer rodizio de equipamentos na sua mesa ao fim de cada etapa do processo, que se realize um treinamento com os responsáveis pelo processo de retirada de pontas de linha, fazendo com que se tenha mais 
atenção e assim não prejudique a seguinte atividade e ainda se recomendou a instrução dos funcionários quanto a distrações como TV e conversas paralelas.

\section{Considerações finais}

Tendo em vista a necessidade das empresas da indústria têxtil situadas no agreste de Pernambuco e regiões próximas em se estabelecer no mercado e conseguir vantagem competitiva, evidenciasse a necessidade de se fazer a racionalização do trabalho entendendo da melhor forma possível os processos envolvidos em sua produção.

Desta forma, a partir da identificação das dificuldades dessas organizações com relação a implementação de técnicas que resultem dados que meçam seu desempenho e auxiliem na identificação gargalos que venham a ser prejudiciais a sua produtividade, o presente trabalho teve como objetivo a implementação dos conhecimentos de Engenharia de Métodos por meio de um estudo de caso de forma a fornecer informações uteis para a tomada de decisão destas empresas.

Conclui-se, portanto, que o estudo realizado atingiu seu objetivo sendo de grande importância para que a empresa tivesse em mãos informações sobre seu desempenho, facilitando assim suas decisões de ordem gerencial e podendo assim obter melhoria em seu desempenho e consequentemente na sua produtividade por meio da implementação das propostas aqui apresentadas.

\section{REFERÊNCIAS}

ADDIPER. Governo de Pernambuco anuncia investimento de $\mathrm{R} \$ 100$ milhões em nova indústria têxtil no interior. Disponível em: <http://www.addiper.pe.gov.br/index.php/governo-de-pernambuco-anunciainvestimento-de-r-100-milhoes-em-nova-industria-textil-no-interior/>. Acesso em: 08 fev. 2020.

AGRESTETEX. Entenda a influência do polo têxtil no Agreste Pernambucano. Disponível em: <https://agrestetex.fcem.com.br/entenda-a-influencia-do-polo-textil-no-agreste-pernambucano/>. Acesso em: 08 fev. 2020 .

BARNES, Ralph M.. ESTUDO DE MOVIMENTOS E DE TEMPOS: PROJETO E MEDIDA DO TRABALHO. 6. ed. São Paulo: Editora Edgard Blucher Ltda., 1977. 648 p.

COOPER, D. R.; SCHINDLER, P. S. Métodos de Pesquisa em Administração. 10. ed. Porto Alegre: Bookman, 2011.

FUJITA, Renata Mayumi Lopes; JORENTE, Maria José. A Indústria Têxtil no Brasil: uma perspectiva histórica e cultural. Revista Modapalavra E-periódico, Santa Catarina, v. 8, n. 15, p.153-174, jul. 2015. Disponível em: <https://www.redalyc.org/pdf/5140/514051496008.pdf>. Acesso em: 06 fev. 2020. 
KON, Anita; COAN, Durval Calegari. TRANSFORMAÇÕES DA INDÚSTRIA TÊXTIL BRASILEIRA: A TRANSIÇÃO PARA A MODERNIZAÇÃO. Revista de Economia Mackenzie, São Paulo, v. 3, n. 3, p.12-34, 25 jun. 2009. Disponível em: <http://editorarevistas.mackenzie.br/index.php/rem/article/view/774>. Acesso em: 06 fev. 2020.

LIMA, Adrilene Gonçalves de; SILVA, Geobervagner Albano da. Aplicações De Técnicas Da Engenharia De Métodos Em Uma Lanchonete Universitária: Um Estudo De Caso.. In: Anais do VII Simpósio de Engenharia de Produção - SIMEP. Anais...Montes Claros(MG) FASA, 2019. Disponível em:

$<$ https//www.even3.com.br/anais/7simep/141601-APLICACOES-DE-TECNICAS-DA-ENGENHARIA-DEMETODOS-EM-UMA-LANCHONETE-UNIVERSITARIA--UM-ESTUDO-DE-CASO>.

MOURA, Cassiano Rodrigues; CÍRICO, Elvis. DESENVOLVIMENTO DE UMA MATRIZ DE

CENTRALIZAÇÃO PARA AUMENTO DE PRODUTIVIDADE EM UMA EMPRESA TÊXTIL.. In: Anais do VII Simpósio de Engenharia de Produção - SIMEP. Anais...Montes Claros(MG) FASA, 2019.

SILVA, E. L.; MENEZES, E. M. Metodologia da pesquisa e elaboração de dissertação. 4. ed. Florianópolis: UFSC, 2005.

YIN, R. K. Estudo de caso: planejamento e métodos. 2.ed. Porto Alegre: Bookman, 2001

PEINADO, Jurandir; GRAEML, Alexandre Reis. Administração da Produção: Operações Industriais e de Serviços. 2. ed. Curitiba: Unicenp, 2007. 747 p. 